the temperature increases (see Table IV) indicates, then, an increase in the relative order of the water molecules upon leaving the environment of other solvent molecules and entering the electrostatic fields of the ions.

The curves in Figure 9 were constructed from results calculated by using eq 5 to evaluate $\log K_{\mathrm{sp}}$ at several constant ionic strengths up to $6 \mathrm{~m}$ and at temperatures up to $300^{\circ}$. These solubility products, each at constant $I$, were considered to show the same temperature dependence as $\ln K_{\mathrm{sp}}{ }^{\circ}$ in eq 10. After sets of the four parameters for several given ionic strengths were determined, values for $\Delta G^{\prime}, \Delta H^{\prime}, \Delta S^{\prime}$, and $\Delta C_{\mathbf{p}}^{\prime}$ (which correspond to the changes in the thermodynamic quantities when 1 mole of calcium hydroxide solid dissolves in a solution of ionic strength $I$ ) were calculated in the same manner used in the calculation of the thermodynamic quantities for the standard state (zero ionic strength). The curves at $350^{\circ}$ represent extrapolated results since the experimental results at this temperature appear inconsistent with those at the lower temperatures. The dashed curve for the average $\Delta C^{\prime}{ }_{\mathrm{p}}$ from 0 to $350^{\circ}$, shown in Figure 9 , is the arithmetic mean for this temperature range.

Acknowledgment. The authors express their gratitude to Professors John E. Ricci, New York University, and James W. Cobble, Purdue University, for their helpful discussions.

\title{
The Reduced Equation of State of Argon and Xenon
}

\author{
by I. Crivelli and F. Danon ${ }^{1}$ \\ Facultad de Ciencias Exactas y Naturales, University of Buenos Aires, Buenos Aires, Argentina \\ (Received January 31, 1967)
}

\begin{abstract}
A comparison of the compressibility factors of argon and xenon reduced in terms of the Kihara intermolecular core model potential is presented. It is found that correspondingstates behavior is better obeyed by using the Kihara rather than the Lennard-Jones potential. The results of this paper indicate that the assumption of the pairwise additivity of the potential seems to be valid within the range covered by the presently available experimental data.
\end{abstract}

In a recent article, ${ }^{2}$ we showed that the second virial coefficient and the viscosity coefficient at low pressures of argon, krypton, and xenon can be correlated on a corresponding states basis by the use of the Kihara core model intermolecular potential $\Phi$ as given by

$$
\Phi=\epsilon_{\mathrm{K}}\left[\left(\frac{\rho_{0}}{\rho}\right)^{12}-2\left(\frac{\rho_{0}}{\rho}\right)^{6}\right]
$$

where $\epsilon_{\mathrm{K}}$ is the minimum of the potential which occurs at $\rho_{0}$. The intermolecular separation $\rho$ is defined as the shortest distance between the outer surface of the cores. This equation is valid for $\rho \geq d$ where $d$ is the diameter of the spherical core. For smaller values of $\rho, \Phi=\infty$. Results obtained by using eq 1 are much more satisfactory than those obtained by using the (12-6) Lennard-Jones (L-J) function.

Levelt ${ }^{3}$ has made a careful study of the compressibility factor of argon and xenon and has shown that the $P V / R T$ isotherms of argon may be transformed into the compressibility isotherms of xenon by multiplying by scale factors, which are ratios of potential param-

(1) To whom correspondence and requests for reprints should be sent at the Department of Chemistry, Massachusetts Institute of Technology, Cambridge, Mass. 02139.

(2) F. Danon and J. C. Rossi, J. Phys. Chem., 70, 942 (1966).

(3) J. M. H. Levelt, Physica, 26, 361 (1960). 
eters usually used to represent variables in reduced form. The best set of scale factors was found to be 1.931 for the temperature and 0.633 for the density, with which a $1 \%$ agreement of the isotherms in the range of overlap was obtained.

Since it had been previously found ${ }^{2}$ that the ratio of reduction factors in terms of the molecular parameters of the Kihara potential were much closer to the ideal ones than those of the Lennard-Jones potential, it could be predicted that if the isotherms of argon and xenon were reduced with our set of parameters they would come much closer to each other than when reduced with L-J parameters. In this article the results of this calculation are presented using the data of Tables I and IV of Levelt's paper, where $P V /$ $R T$ values of argon and xenon are listed in terms of the reduced temperature and the reduced density based on the L-J parameters. For example

$$
T_{\mathrm{LJ}}{ }^{*}=T \frac{k}{\epsilon_{\mathrm{LJ}}} ; \quad T_{\mathrm{K}}{ }^{*}=T_{\mathrm{LJ}} * \frac{\epsilon_{\mathrm{LJ}}}{\epsilon_{\mathrm{K}}}
$$

where $T_{\mathrm{LJ}}{ }^{*}$ and $T_{\mathrm{K}}{ }^{*}$ are the reduced temperatures in terms of the Lennard-Jones and Kihara potential parameters $\epsilon_{\mathrm{LJ}}$ and $\epsilon_{\mathrm{K}}$, respectively, and $k$ is the Boltzmann constant. Numerical values for the ratio of the $\epsilon$ 's are 0.868 for argon and 0.847 for xenon. Correspondingly, we obtain for the reduced density $d^{*}$

$$
d_{\mathrm{LJ}} *=\frac{N \sigma_{\mathrm{LJ}}{ }^{3}}{V_{\mathrm{m}}} ; \quad d_{\mathrm{K}}{ }^{*}=d_{\mathrm{LJ}} * \frac{\sigma_{\mathrm{K}}{ }^{3}}{\sigma_{\mathrm{LJ}}{ }^{3}}
$$

where $\sigma_{\mathrm{LJ}}$ and $\sigma_{\mathrm{K}}$ are the distances between molecular centers when the potentials are zero. $V_{\mathrm{m}}$ is the molar volume, and $N$ is Avogadro's number. Numerical values for the ratio of the $\sigma^{3} \mathrm{~s}$ are 0.978 for argon and 0.900 for xenon. The collision diameter $\sigma$ is obtained from $\sigma=2^{-1 / s} \rho_{0}+d$, where $\rho_{0}$ is the potential parameter in eq 1. The diameter of the spherical core $d$ is not an extra, adjustable parameter but is independently fixed ${ }^{4 a}$ by the simple relationship $d=0.08 \rho_{0}$, so that $\sigma=\rho_{0}\left(2^{-1 / 6}+0.08\right)=0.971 \rho_{0}$. We thus obtained a two-parameter potential function as required by the theory of corresponding states. ${ }^{4 b}$ The molecular parameters we used are shown in Table I. They have been obtained by simultaneous fitting of both

Table I: Parameters for the Kihara Potential

\begin{tabular}{lccc} 
& $\begin{array}{c}e / k, \\
{ }^{\circ} \mathrm{K}\end{array}$ & $\stackrel{a}{\mathrm{~A}}$ & $\stackrel{d,}{\mathrm{~A}}$ \\
Argon & 138.0 & 3.381 & 0.279 \\
Xenon & 266.0 & 3.932 & 0.324 \\
\hline
\end{tabular}

second virial coefficient and viscosity coefficient. ${ }^{5}$ Values of the parameters for xenon differ somewhat from those of ref 5 because recent viscosity measurements by Rigby and Smith $^{6}$ have been included in determining $\epsilon / k$ and $\sigma$; Lennard-Jones parameters are, of course, the same ones Levelt used.

We have performed a two-variable polynomial interpolation on the xenon data thus obtaining the $P V / R T$ surface in the $\left(P V / R T, T_{\mathbf{K}}{ }^{*}, d_{\mathrm{K}}{ }^{*}\right)$ space by automatic computation. We used the least-squares program 1007 on the Mercury computer of this university. The resulting polynomial $Z\left(T_{\mathrm{K}}{ }^{*}, d_{\mathrm{K}}{ }^{*}\right)^{7}$ is of the sixth degree in $d^{*}$ and the fifth degree in $T^{*}$, six being the maximum power of any term. This function gives a sum of the squares of the differences between the calculated and the observed value of $P V / R T$ of xenon of $1.73 \times 10^{-4}$ for the 136 data points used. We then calculated from the polynomial $Z$ the values of $P V / R T$ of xenon at values of $T_{\mathrm{K}}{ }^{*}$ and $d_{\mathrm{K}}{ }^{*}$ corresponding to those available from the actual measurements on argon. These values of $P V / R T$ of xenon and argon for the same values of the reduced variables illustrate how well corresponding states are followed. Results of the calculations are shown in Table II where we list the differences ( $P V / R T$ of argon $-P V / R T$ of xenon) reduced in terms of the Kihara potential parameters. Differences in terms of the Lennard-Jones parameters as taken from Levelt's paper are also included for comparison in Table III.

It can be observed that the differences are now significantly reduced in almost the entire range of overlap which extends from 1.17 to 1.56 in reduced temperature and from 0.098 to 0.685 in reduced density. The sum of the squares of the differences is $4.15 \times 10^{-3}$ (standard deviation $6.32 \times 10^{-3}$ ) for the Kihara potential and 1.437 (standard deviation 0.126) for the Lennard-Jones potential. In Table II there are two more columns than in Table III, i.e., reduced densities 0.562 and 0.611 . These correspond to densities 0.575 and 0.625 , respectively, for the L-J case and do not appear in Table III because there are no experimental values of $P V / R T$ for xenon reported for these densities. These results were in fact expected from a comparison of the values of the scale factors. The scale factor for temperature is simply the ratio $\epsilon_{\mathrm{K}}$ of xenon/ $\epsilon_{\mathrm{K}}$ of argon. The scale factor for density is ( $\sigma_{\mathrm{K}}$ of argon $)^{3} /\left(\sigma_{\mathrm{K}}\right.$ of xenon) ${ }^{3} \times V_{\mathrm{s}}$ of xenon $/ V_{\mathrm{s}}$ of argon where $V_{\mathrm{s}}$ is the

(4) (a) F. Danon and K. S. Pitzer, J. Chem. Phys., 36, 425 (1962); (b) K. S. Pitzer, ibid., 7, 583 (1939).

(5) J. C. Rossi and F. Danon, Discussions Faraday Soc., 40, 97 (1965).

(6) M. Rigby and E. B. Smith, Trans. Faraday Soc., 62, 54 (1966). (7) Numerical values of the coefficients will be furnished on request. 
Table II: Differences of Compressibility Factors (PV/RT of Argon - PV/RT of Xenon);

Reduction with Kihara Molecular Parameters

\begin{tabular}{|c|c|c|c|c|c|c|c|c|}
\hline$T_{\mathrm{K}^{*}}$ & 0.098 & 0,147 & 0.196 & 0.2445 & 0.293 & 0.318 & 0,342 & 0.391 \\
\hline 1.17 & 0.0068 & 0.0100 & 0.0095 & 0.0076 & 0.0069 & 0.0077 & 0.0078 & 0.0087 \\
\hline 1.215 & 0.0063 & 0.0088 & 0.0087 & 0.0071 & 0.0060 & 0.0062 & 0.0060 & 0.0062 \\
\hline 1.26 & 0.0043 & 0.0054 & 0.0051 & 0.0033 & 0.0017 & 0.0015 & 0.0009 & 0.0004 \\
\hline 1.30 & 0.0045 & 0.0054 & 0.0057 & 0.0046 & 0.0035 & 0.0033 & 0.0029 & 0.0025 \\
\hline 1.39 & 0.0030 & 0.0028 & 0.0034 & 0.0031 & 0.0026 & 0.0026 & 0.0023 & 0.0019 \\
\hline 1.475 & 0.0027 & 0.0023 & 0.0035 & 0.0041 & 0.0043 & 0.0043 & 0.0043 & 0.0042 \\
\hline \multirow[t]{2}{*}{1.56} & 0.0010 & -0.0003 & 0.0005 & 0.0008 & 0.0005 & 0.0000 & -0.0004 & -0.0015 \\
\hline & 0.440 & 0.488 & 0.538 & 0.562 & 0.587 & 0.611 & 0.636 & \\
\hline 1.17 & 0.0091 & 0.0091 & 0.0098 & 0.0121 & 0.0119 & 0.0164 & 0.0165 & \\
\hline 1.215 & 0.0064 & 0.0067 & 0.0078 & 0.0104 & 0.0102 & 0.0148 & 0.0144 & \\
\hline 1.26 & -0.0001 & -0.0004 & 0.0003 & 0.0026 & 0.0022 & 0.0063 & 0.0055 & \\
\hline 1.30 & 0.0024 & 0.0025 & 0.0036 & 0.0062 & 0.0058 & 0.0100 & 0.0089 & \\
\hline 1.39 & 0.0015 & 0.0013 & 0.0020 & 0.0046 & 0.0036 & 0.0074 & 0.0057 & \\
\hline 1.475 & 0.0039 & 0.0040 & 0.0050 & 0.0077 & 0.0066 & 0.0105 & 0.0085 & \\
\hline 1.56 & -0.0024 & -0.0026 & -0.0020 & 0.0006 & -0.0006 & 0.0032 & 0.0014 & \\
\hline
\end{tabular}

Table III: Differences of Compressibility Factors ( $P V / R T$ of Argon $-P V / R T$ of Xenon);

Reduction with Lennard-Jones Molecular Parameters

\begin{tabular}{|c|c|c|c|c|c|c|c|}
\hline$T_{\mathrm{LJ}} *$ & 0.100 & 0.150 & 0.200 & 0.250 & 0.300 & 0.325 & 0.350 \\
\hline 1.35 & -0.0013 & 0.0000 & 0.0046 & 0.0064 & 0.0116 & 0.0149 & 0.0186 \\
\hline 1.40 & -0.0010 & 0.0004 & 0.0033 & 0.0074 & 0.0130 & 0.0167 & 0.0211 \\
\hline 1.45 & -0.0005 & 0.0009 & 0.0039 & 0.0083 & 0.0145 & 0.0185 & 0.0234 \\
\hline 1.50 & -0.0003 & 0.0013 & 0.0046 & 0.0093 & 0.0160 & 0.0205 & 0.0258 \\
\hline 1.60 & 0.0003 & 0.0024 & 0.0060 & 0.0113 & 0.0188 & 0.0240 & 0.0301 \\
\hline 1.70 & 0.0010 & 0.0037 & 0.0076 & 0.0135 & 0.0218 & 0.0274 & 0.0340 \\
\hline \multirow[t]{2}{*}{1.80} & 0.0017 & 0.0046 & 0.0090 & 0.0155 & 0.0246 & 0.0306 & 0.0376 \\
\hline & 0.400 & 0.450 & 0.500 & 0.550 & 0.600 & 0.650 & \\
\hline 1.35 & 0.0298 & 0.0484 & 0.0798 & 0.1307 & 0.2075 & 0.3187 & \\
\hline 1.40 & 0.0338 & 0.0541 & 0.0869 & 0.1387 & 0.2152 & 0.3247 & \\
\hline 1.45 & 0.0374 & 0.0590 & 0.0929 & 0.1452 & 0.2216 & 0.3296 & \\
\hline 1.50 & 0.0407 & 0.0633 & 0.0981 & 0.1509 & 0.2270 & 0.3335 & \\
\hline 1.60 & 0.0467 & 0.0709 & 0.1071 & 0.1603 & 0.2357 & 0.3392 & \\
\hline 1.70 & 0.0518 & 0.0773 & 0.1144 & 0.1680 & 0.2424 & 0.3433 & \\
\hline 1.80 & 0.0562 & 0.0826 & 0.1204 & 0.1739 & 0.2473 & 0.3463 & \\
\hline
\end{tabular}

Table IV: Values of the Scale Factors for the Isotherms

\begin{tabular}{lccc} 
& $\begin{array}{c}\text { Levelt } \\
\text { L-J }\end{array}$ & $\begin{array}{c}\text { Present } \\
\text { work }\end{array}$ & Ideal \\
Temperature & 1.881 & 1.927 & 1.931 \\
Density & 0.582 & 0.631 & 0.633 \\
\hline
\end{tabular}

standard molar volume of the gas as calculated from Levelt's data. ${ }^{3}$ Numerical values of the scale factors are shown in Table IV.

The fact that the Lennard-Jones potential does not provide an adequate description of the molecular field of the rare gases seems by now well established..$^{5.8}$ The Kihara potential, on the other hand, gives much more satisfactory results in the calculation of both equilibrium and transport properties of the rare gases..$^{5,9}$ Both the nature of the potential and the assumption of pairwise additivity are determining factors in satis-

(8) T. Kihara, Rev. Mod. Phys., 25, 831 (1955); W. B. Brown and J. S. Rowlinson, Mol. Phys., 3, 35 (1960); R. Munn, J. Chem. Phys., 40, 1439 (1964).

(9) A. E. Sherwood and J. M. Prausnitz, ibid., 41, 413 (1964); J. A. Barker, W. Fock, and F. Smith, Phys. Fluids, 7, 897 (1964). 
fying corresponding states behavior. If we accept that the Kihara core model gives an adequate representation of the molecular interactions of the heavy rare gases, as a growing body of evidence now indicates, then the results of this paper indicate that the assump- tion of the additivity of the potential seems to be valid for thermodynamic calculations of the fluid phase within the range so far studied.

Acknowledgment. The authors sincerely thank Professor I. Amdur for a critical reading of the manuscript.

\title{
Photoenolization of Biacetyl
}

\author{
by Jacques Lemaire ${ }^{1}$ \\ Department of Chemistry, The University of Texas, Austin, Texas 78712 (Received February 2, 1966)
}

\begin{abstract}
Irradiation of biacetyl either in hexane or in aqueous solution below $3100 \mathrm{~A}$ (to give the second excited singlet state) gives a product which is probably an enol. Biacetyl in a cis form could give an enol which would be stabilized by internal hydrogen bonding. The quantum yield of enol formation is about 0.12. In the vapor phase a similar product is formed. At $4358 \mathrm{~A}$, enol formation at room temperature either in solution or in the vapor state is essentially zero but is appreciable at $100^{\circ}$. The compound tentatively identified as the enol inhibits both the phosphorescence and the decomposition upon exposure to wavelengths which give the first excited singlet state. Since oxygen does not quench the fluorescence of biacetyl but quenches both the phosphorescence and the formation of the enol, it is suggested that the enol arises from the triplet state of biacetyl. If the triplet state is a precursor for enol formation, the data suggest that an activation energy is necessary and hence that high vibrational levels of the triplet state are involved.
\end{abstract}

\section{Introduction}

The photochemistry of biacetyl has received much attention both in the vapor phase and in solution. ${ }^{2 a}$ The results of Porter ${ }^{2 b}$ and those of Bäckström and Sandros ${ }^{2 c}$ indicate that all initially formed singlet molecules at $4358 \mathrm{~A}$ which do not either dissociate or fluoresce cross over to the triplet state. Presently known processes do not account for all of the tripletstate molecules.

Fluorescence from biacetyl when excited to the first singlet state either in the liquid or in the gas phase is very small, with a quantum yield of about $0.0025 .^{3}$ Thus, crossover to the triplet state at $4358 \mathrm{~A}$ occurs with a yield greater than 0.99 . Phosphorescence from the triplet state has a yield of about 0.15. Decomposition at this wavelength is negligible at room tem- perature, although there is some dissociation which results from interaction between two excited molecules. ${ }^{4}$ The dissociation yield increases rapidly with increase in temperature.

Some unidentified product from biacetyl at $4358 \mathrm{~A}$

(1) Address all correspondence to the author at the Ecole Nationale Superieure des Industries Chimiques, 1 Rue Grandville, Nancy, France.

(2) (a) W. A. Noyes, Jr., G. B. Porter, and J. E. Jolley, Chem. Rev., 56, 49 (1956); (b) G. B. Porter, J. Chem. Phys., 32, 1587 (1960); (c) H. J. L. Bäckström and K. Sandros, Acta Chem. Scand., 14, 48 (1960).

(3) G. M. Almy and P. R. Gillette, J. Chem. Phys., 11, 188 (1943) The fiuorescent yield in dilute solution may be as high as 0.01 . F. Wilkinson and J. T. Dubois, ibid., 39, 377 (1963).

(4) W. A. Noyes, Jr., W. A. Mulac, and M. S. Matheson, ibid., 36, $880(1962)$; G. F. Sheats and W. A. Noyes, Jr., J. Am. Chem. Soc., $77,4532(1955)$ 